\title{
RAIRO-RO
}

\section{La division Aide à la décision-systèmes de gestion}

Revue française d'automatique, d'informatique et de recherche opérationnelle. Recherche opérationnelle, tome 9, $\mathrm{n}^{\circ} \mathrm{V} 2$ (1975), p. 126.

<http://www.numdam.org/item?id=RO_1975_9_2_126_0>

(C) AFCET, 1975, tous droits réservés.

L'accès aux archives de la revue « Revue française d'automatique, d'informatique et de recherche opérationnelle. Recherche opérationnelle » implique l'accord avec les conditions générales d'utilisation (http://www.numdam.org/ legal.php). Toute utilisation commerciale ou impression systématique est constitutive d'une infraction pénale. Toute copie ou impression de ce fichier doit contenir la présente mention de copyright.

\section{Numdam}

Article numérisé dans le cadre du programme

Numérisation de documents anciens mathématiques

http://www.numdam.org/ 


\section{LA DIVISION \\ AIDE A LA DECISION-SYSTEMES DE GESTION}

(Recherche Opérationnelle et Automatique de Gestion) de l'A.F.C.E.T.

a décidé d'attribuer deux prix annuels de Recherche Opérationnelle à des étudiants des Universités ou des Grandes Écoles. L'un est destiné aux élèves de Second Cycle ou équivalent (moins de quatre années d'études, ou cycle normal des Grandes Écoles), l'autre aux élèves de troisième cycle ou équivalent.

Les candidats devront être sélectionnés par leur professeur, qui ne pourra présenter plus de deux personnes.

Pour concourir, le candidat devra envoyer, en triple exemplaire, son travail, résumé si nécessaire, en moins de 300 lignes dactylographiées au Secrétariat de l'A.F.C.E.T., accompagné d'une lettre faisant acte de candidature, de la recommandation de son professeur qui devra indiquer pour lequel des deux prix le candidat concourt.

Le jury désigné par la division décernera deux ou trois prix dans chacune des deux sections. Les travaux des premiers prix seront publiés dans la Revue Verte, avec mention complète, en plus du nom de l'auteur, de son École ou Université et du nom de son Professeur.

Pour l'année 1975, les dossiers de candidatures doivent parvenir au Secrétariat de l'A.F.C.E.T.

156, boulevard Pereire, 75017 Paris

avant le 15 octobre 1975

avec la mention «PRIX DE RECHERCHE OPÉRATIONNELLE de l'A.F.C.E.T. ". 